\title{
Systemic Bevacizumab for Recurrent Respiratory Papillomatosis
}

\author{
Yiressy Izaguirre Baday ${ }^{1}$, Julina Ongkasuwan ${ }^{1}$, and Rajkumar Venkatramani² \\ ${ }^{1}$ Baylor College of Medicine \\ ${ }^{2}$ Texas Children's Hospital
}

June 8, 2020

\begin{abstract}
Recurrent respiratory papillomatosis (RRP) is a benign tumor of the respiratory tract associated with human papillomavirus types 6 and 11. Patients undergo multiple surgical debridements for management of rapidly growing papilloma. Adjuvant treatment options for management of RRP in children are often ineffective and do not decrease the need for repeated surgical debridement. We report on three patients initially treated with $10 \mathrm{mg} / \mathrm{kg}$ systemic bevacizumab every 4 weeks. All patients had improvement in voice and reduced need for surgical debridement. The interval between bevacizumab doses was gradually increased to every 8-12 weeks. Adverse events included mild proteinuria and self-resolving epistaxis.
\end{abstract}

\section{Introduction}

Recurrent Respiratory Papillomatosis (RRP) is a benign neoplasm of the respiratory tract associated with human papillomavirus (HPV) 6 and $11^{1}$. Incidence is estimated at 4.3 in $100000^{2}$. Patients present with hoarseness ${ }^{1-4}$, signs of airway obstruction ${ }^{3,4}$, and rarely malignant transformation may occur ${ }^{3}$. Surgical debridement is the mainstay of symptom management. Patients often undergo multiple surgical procedures ${ }^{3,4}$ that can result in complications such as vocal fold scar and laryngeal stenosis ${ }^{5}$.

Several adjuvant treatment options have been proposed to decrease the need for repeated surgical removal of tumors including intralesional cidofovir and bevacizumab. Mohr et al, 2014 first reported good or partial improvement with the use of systemic bevacizumab in five patients with RRP ${ }^{4}$. In 2016, Zur and Fox described use of systemic bevacizumab in a 12 year old female with $\mathrm{RRP}^{3}$. In this case series we describe our experience treating three patients with juvenile onset RRP with systemic bevacizumab.

\section{Methods}

Exempt status was obtained from Baylor College of Medicine's Institutional Review Board. Charts were retrospectively reviewed.

\section{Case Report}

Patient 1

Ten-year-old male who presented at 11 months of age with dyspnea, snoring and apneas. Laryngoscopy revealed extensive papilloma involving the supraglottis, glottis, and proximal trachea. Pathology confirmed papilloma with changes consistent with HPV effect. The patient underwent 75 surgical debridement procedures by 10 years of age with an average of 6.6 weeks between procedures. Six injections of intralesional cidofovir were tried without significant improvement.

Intravenous bevacizumab $10 \mathrm{mg} / \mathrm{kg}$ every 4 weeks was started in February 2019. The interval between treatments was four weeks between cycles 1-6, and was gradually increased to eight weeks from cycle 11 onwards. The patient has received 12 cycles so far. Voice quality improved after the first cycle with significant 
improvement reported after cycle 6 . Laryngoscopy performed after 3 and 6 cycles showed minimal disease (Fig. 1). The only side effect observed was asymptomatic proteinuria which has remained stable since baseline examination.

Patient 2

Five-year-old male who presented at 3 years of age with hoarseness, stridor, and snoring since infancy. Laryngoscopy showed presence of bulky supraglottic and glottis papilloma. He underwent 15 debridement procedures every 8.6 weeks before initiation of systemic bevacizumab. He received four intralesional bevacizumab injections without significant improvement.

Intravenous bevacizumab $10 \mathrm{mg} / \mathrm{kg}$ was started in July 2018. Infusions were given every four weeks during cycles 1-4, with a gradual increase to eight-week intervals between cycles 7-9, and every 12 weeks from cycle 9 onward. Patient has received 13 cycles so far. Laryngoscopy performed during the first 11 cycles showed minimal or no papilloma (Fig. 2). Formal speech evaluation after five cycles showed improvement in voice. Self-limited epistaxis attributed to allergic rhinitis was the only side effect reported. No abnormalities in growth plate was noted in X ray knee obtained after 11 cycles. Eight weeks after receiving cycle 11 bevacizumab, patient developed worsening voice quality. Flexible nasolaryngoscopy showed recurrence of papillomas and decision was made to resume bevacizumab at 8 -week intervals.

Patient 3

Eight-year-old female who was diagnosed with RRP at eight months of age. Before starting IV bevacizumab the patient underwent 35 procedures at our institution on average every 8.2 weeks. She developed worsening hoarseness and stridor prior to debridement procedures. The papilloma burden affected patient's activities of daily living due to dyspnea with exercise, snoring and mockery at school. She underwent four intralesional bevacizumab injections without significant improvement.

Patient started $10 \mathrm{mg} / \mathrm{kg}$ IV bevacizumab in October of 2018. Interval between cycles was four weeks between cycles 1-4 and gradually increased to every 8 weeks by cycle 10 . Parents noted improvement in voice and exercise tolerance after one cycle. Laryngoscopy examinations after starting bevacizumab showed small or no papillomas. After eight cycles, speech evaluation showed moderate symptoms of vocal disruption including a rough, raspy voice without impact on function, with parents reporting voice as being at its best. Only side effects reported was self-resolving epistaxis after the first cycle.

\section{Discussion}

In our experience, administration of systemic bevacizumab for RRP in children results in rapid improvement in voice quality, symptom relief and reduced need for surgical debridement. The treatment was well tolerated and side effects were minimal and included mild proteinuria and brief epistaxis. This is not surprising as the dose and frequency we use is lower than that used for treating children with cancer such as brain tumors. Although bevacizumab controls the growth of papillomas, it is unlikely that it will be curative. This raises concerns about long term use of bevacizumab in young children. The possible long-term effects of bevacizumab has to be balanced against the risks of repeated surgical procedures, anesthesia and lower voice quality. Our approach is to use the minimum dose and frequency necessary to avoid surgical debridements. To this end, we rapidly increase the interval between doses until papillomas recur or symptoms return.

The use of adjuvant therapies in the management of RRP has had mixed results ${ }^{1-5}$. A 2003 case series on the use of intralesional cidofovir on four children with RRP had heterogenous results with one of the patients achieving complete remission for 15 months $^{5}$. A 2013 case series that evaluated the effects of three injections of intralesional bevacizumab in 10 children with RRP saw a median surgical procedure time increase of 5.9 weeks ${ }^{1}$. All three of our patients did not achieve significant benefit from use of intralesional cidofovir or bevacizumab.

Our case series is limited by a short follow up of patients which lessens our ability to determine long term efficacy as well as late side effects. Our patients lacked standardized surveys to assess symptom severity before 
and after starting treatment. HPV typing was not assessed which limited our ability to draw conclusions about the relationship between treatment response and HPV type.

Conflict of interest: Julina Ongkasuwan receives royalties from Springer and Elsevier. There are no other conflicts of interests to report.

Acknowledgements: There are no acknowledgements.

References

1. Rogers DJ, Ojha S, Maurer R, Hartnick CJ. Use of Adjuvant Intralesional Bevacizumab for Aggressive Respiratory Papillomatosis in Children. JAMA Otolaryngol Neck Surg . 2013;139(5):496-501. doi:10.1001/jamaoto.2013.1810

2. Derkay CS. Task force on recurrent respiratory papillomas. A preliminary report. Arch Otolaryngol Head Neck Surg . 1995;121(12):1386-1391. doi:10.1001/archotol.1995.01890120044008

3. Zur KB, Fox E. Bevacizumab chemotherapy for management of pulmonary and laryngotracheal papillomatosis in a child. The Laryngoscope . 2017;127(7):1538-1542. doi:10.1002/lary.26450

4. Mohr M, Schliemann C, Biermann C, et al. Rapid response to systemic bevacizumab therapy in recurrent respiratory papillomatosis. Oncol Lett . 2014;8(5):1912-1918. doi:10.3892/ol.2014.2486

5. Milczuk HA. Intralesional cidofovir for the treatment of severe juvenile recurrent respiratory papillomatosis: long-term results in 4 children. Otolaryngol Head Neck Surg . 2003;128(6):788-794. doi:10.1016/S0194$5998(03) 00259-6$

Figure Legends:

FIGURE 1 Laryngoscopy Before and After Bevacizumab in Patient 1. A-C show images from the last laryngoscopy performed before Bevacizumab. Figure A shows papilloma in epiglottis, figure B in glottis and figure $\mathrm{C}$ in trachea. Figures $\mathrm{D}$ to $\mathrm{F}$ show the first post bevacizumab laryngoscopy with minor papilloma laryngeal side of epiglottis (D), vocal folds (E) and a clear trachea. Figures G-I shows the most recent laryngoscopy showing minor papilloma in vocal folds $(\mathrm{G})$ with persistently papilloma free trachea (H and $\mathrm{I}$ ).

FIGURE 2 Laryngoscopy Before and After Bevacizumab in Patient 2. Figure A shows last laryngoscopy performed before procedure which shows bulky papilloma along entire length of vocal folds. Figures BD show first laryngoscopy after bevacizumab which shows minimal papillomas on vocal folds. Figure E shows laryngoscopy performed after 4 cycles which does not show any papillomas. Picture F shows flexible nasolaryngoscopy obtained after 8 cycles of bevacizumab which also shows no papillomas. 

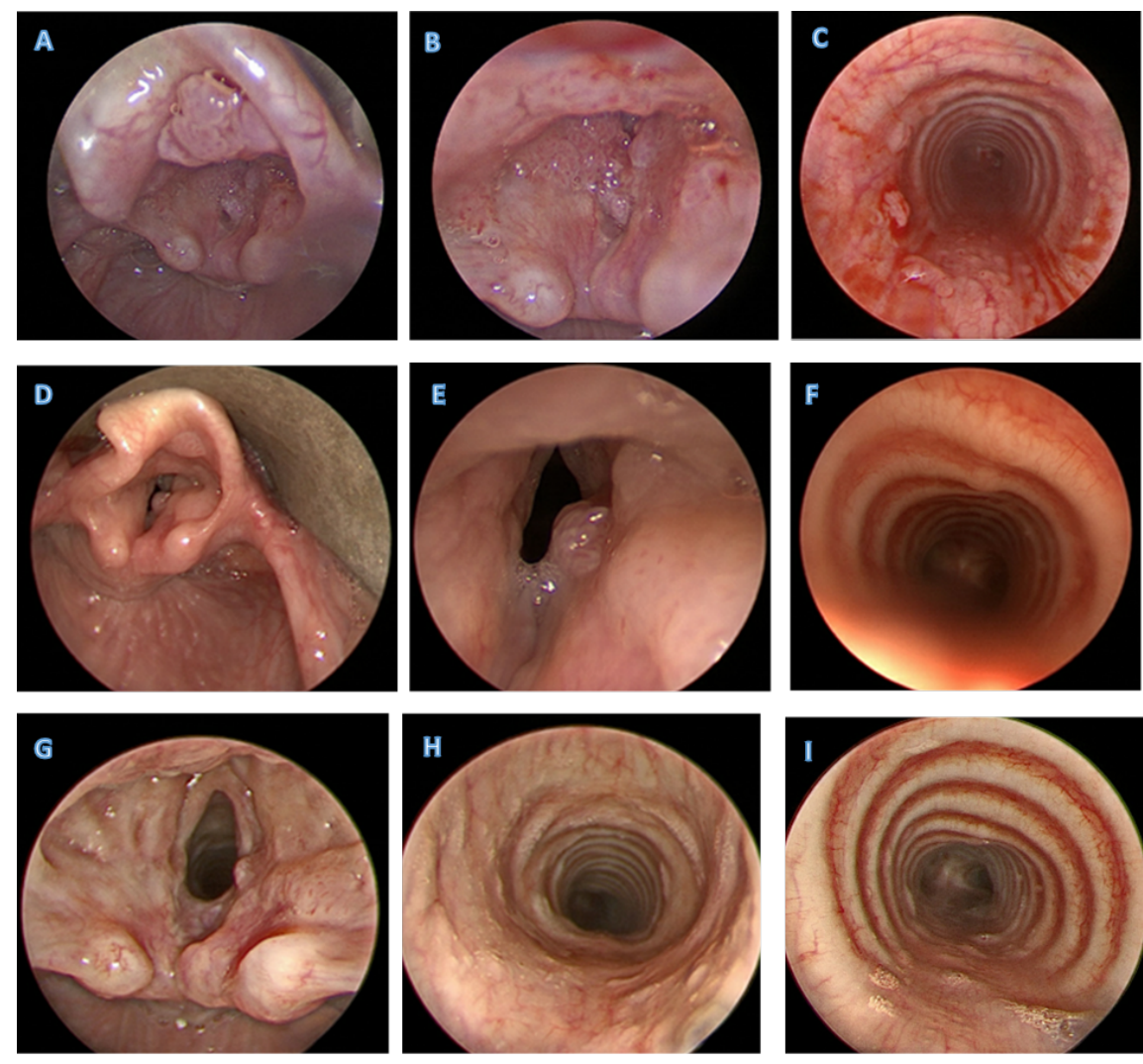

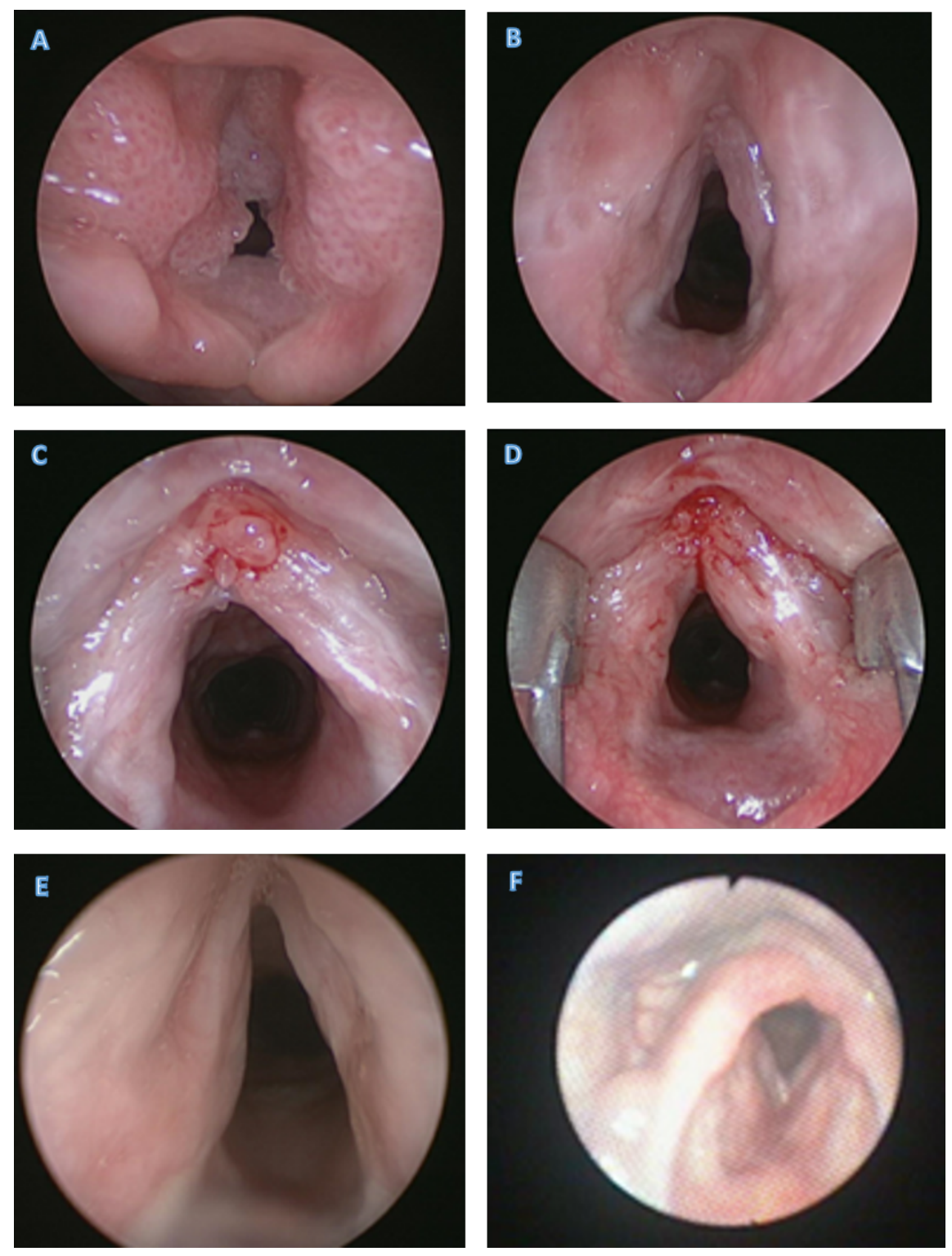